\title{
PECULIARITIES OF THE ORBIT MORPHOGENESIS AT AN EARLY PERIOD OF HUMAN ONTOGENESIS
}

DOI:10.36740/WLek202102101

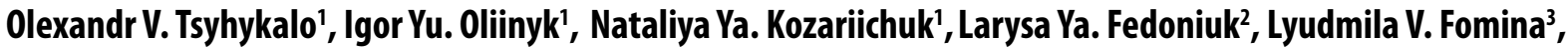 \\ Olha L. Ocheretna ${ }^{3}$, Viktoriia V. Piliponova ${ }^{3}$ \\ 'BUKOVINIAN STATE MEDICAL UNIVERSITY, CHERNIVTSI, UKRAINE \\ 2. HORBACHEVSKY TERNOPIL NATIONAL MEDICAL UNIVERSITY, TERNOPIL, UKRAINE \\ ${ }^{3}$ VINNITSYA MEMORIAL PYROGOV NATIONAL MEDICAL UNIVERSITY, VINNYTSIA, UKRAINE
}

\begin{abstract}
The aim: To clarify the sources and determine the chronological sequence of the germs of anatomical structures of the orbit at the early period of human ontogenesis.

Materials and methods: Using a complex of methods of morphological examination (morphometry, microscopy, three-dimensional computer reconstruction and statistical analysis) 30 series of consecutive histological sections of human embryos and prefetuses aged from 3 till 8 weeks od IUD (3.0-30.0 mm parietal-coccygeal length (PCL)) were studied. Results: At the $4^{\text {rd }}$ week of IUD the orbital region is represented by the place of close contact of the neuroectoderm of the optic vesicle with the adjacent integumentary ectoderm, as well as the mesenchyme surrounding this place of contact. In embryos of $4.0 \mathrm{~mm} \mathrm{PCL}$, the rudiment of the optic stalk is observed as a result of the transformation of the junction of the area of the eye rudiment with the brain.

Conclusions: 1. The rudiments of the organ of vision (lens placodes) appear in the 4rd week of IUD. At the 5th week of IUD as a result of gradual intussusception of crystalline placodes into the adjacent mesenchyme, lens pit are formed, and then - lens vesicles. 2. Rudiments of the extraocular muscles (except for the inferior oblique muscle) was detected at the end of the 5th week of IUD. The rudiment of the inferior oblique muscle develops from a single mesodermal islet located in the mesenchyme medially and below the eyeball. Simultaneously with the rudiments of the extraokular muscles, the trochlear and abductor nerves are develop and ingrown into the orbit. 3. The development of blood vessels of the orbit occurs from two sources - from the islands of local angiogenesis, which begins in the 5 th week of IUD, and from extraorganic vessels, which can be traced in the form of a vascular network at the end of the 6 th week of IUD. The combination of both sources is observed at the end of the 7th week of IUD. 4. The embryonic period of ontogenesis is the first critical period in the development of the human orbit, due to the formation of the muscles, nerve and vascular structures.
\end{abstract}

KEY WORDS: orbit, embryo, prefwtus, prenatal ontogenesis, human

Wiad Lek. 2021;74(2):179-183

\section{INTRODUCTION}

Finding out the sources, the chronological sequence of the germs and the formation of the structure of the human orbit remains a important task for morphologists. Eye diseases in $85.3 \%$ of cases are congenital or acquired in childhood $[1,2]$. One of the main causes of strabismus, amblyopia, binocular vision disorders, myopia and astigmatism is the pathology of the eyeball muscles [3]. Clinicians have repeatedly emphasized that scientific studies of age-dependet morphological features of the organ of vision are clearly insufficient [4]. In addition, the development and application of new microsurgical operations require a deeper knowledge of the surgical anatomy of the orbit [5]. Anatomical data on the development and formation of topography of the vascular-nervous and muscular structures of the human eyeball are fragmentary and do not give a clear data of the sequence of structural changes during intrauterine development (IUD) $[1,3]$. A comprehensive study of the features of development, formation, topographic and anatomical changes in the structures of the orbit (in particular, the muscles of the eyeball, blood vessels and nerves), the dynamics of their syntopic changes during the erly prenatal period of ontogenesis is important to determine the structure, preconditions and time of occurrence of their congenital malformations $[6,7]$.

\section{THE AIM}

To clarify the sources and determine the chronological sequence of the germs of anatomical structures of the orbit at the early period of human ontogenesis.

\section{MATERIALS AND METHODS}

We have examined 30 series of consecutive histological sections of human embryos and prefetuses aged from 3 till 8 weeks of IUD (3.0-30.0 mm parietal-coccygeal length $(\mathrm{PCL})$ ). The material was obtained and studied at Chernivtsy Regional Pathologists Office (Ukraine) in accordance with bilateral collaboration with the Department of Histology, Cytology and Embryology. In order to visualize necessary structures of the orbit we have used complex of 
methods of morphological investigation: morphometry, microscopy, 3D-reconstruction and statistical analysis. The study was performed in accordance with the provisions of the Declaration of Helsinki on ethical issues of studies conducted with humans (1964-2008). All specimens were obtained from ectopic pregnancies or spontaneous abortions, and no part of the material gave indications of possible malformation. Approval for the study was granted by the Ethics Committee of the Bukovinian State Medical University.

\section{RESULTS}

To development of the orbit region with the organ of vision precedes the process of differentiation of the rudiment of the anterior part of the neural tube from three cerebral vesicles (anterior - forebrain, middle - midbrain and posterior - rhombencephalon) into five due to the separation of the middle and posterior cerebral vesicles. The anterior cerebral vesicles forms the diencephalon and the telencephalon. The stage of the five-vesicles rudiment of the brain is clearly observed at the beginning 3rd weeks of IUD. The sources of the eyeball rudiment are traced at the beginning of the 4rd week of IUD (embryos of 4.0 and $4.3 \mathrm{~mm} \mathrm{PCL}$ ), when the lateral protrusions of the neuroectoderm of the diencephalon (optic vesicles) reach the ectodermal cover of the embryo (Fig. 1). During this period, the orbital region is represented by the place of close contact of the neuroectoderm of the optic vesicle with the adjacent integumentary ectoderm, as well as the mesenchyme surrounding this place of contact.

In embryos of $4.0 \mathrm{~mm}$ PCL, the rudiment of the optic stalk is observed as a result of the transformation of the junction of the area of the eye rudiment with the brain (Fig. 2).

In embryos of 4.0-5.0 mm PCL (4th week of IUD) structural transformations of tissues at the point of contact of the nervous tissue of the eyeball and the ectoderm of the lens placode are observed. Nerve tissue continues to protrude into the adjacent mesenchyme in the direction of the ectoderm, and the ectoderm of the lens placode thickens, invaginates the adjacent neuroectoderm, which leads to the formation of lens pits and the beginning of formation the optic cups (Fig. 3).

The mesenchyme surrounds the vesicles and the optic stalk, and extends from the brain to the ectodermal covering of the cranial part of the embryo. In human embryos of 4.0 and $5.0 \mathrm{~mm}$ PCL (4th week of IUD) observed a condensation of the mesenchyme in the rudiment of the orbital region, it localized around the junction of the optic stalk with the cerebral vesicle. We believe that this condensation of the mesenchyme in the form of a membranous plate, close to the rudiment of the brain, has no direct anatomical relation to the organ of vision, and is the rudiment of the ectomeningeal capsule.

In embryos of 7.0 and $7.5 \mathrm{~mm}$ PCL (5th week of IUD) there is a gradual intussusception of lens placodes into the adjacent mesenchyme, which leads to their transformation into lens pit, and then - in the lens vesicle. The lens vesicles begin to untie from the integumentary epithelium of the head, close and turn into epithelial bodies of round shape, adjacent to the optic vesicles. Their wall is invaginated, as a result of which they gradually turn into double-walled cups. The optic cups are connected to the cavity of the anterior cerebral vesicle by means of the optic stalk. Both optic cups and optic stalks are in the cell mass of the mesenchyme. Subsequently, the lens placoda forms the lens of the eye, while the optic cup forms the retina and other parts of the eye (Fig. 4).

The sources of the extraocular muscles (except for the inferior oblique muscle) was first detected at the end of the 5th week of IUD, when in embryos 7.0-7.5 mm PCL in the adjacent mesenchyme behind the optic cups and around the optic stulks appear the foci of condensation of mesodermal cells of irregular elongated shape (Fig. 5). The rudiment of the inferior oblique muscle develops from a single mesodermal islet located in the mesenchyme medially and below the eyeball.

$3 \mathrm{D}$-reconstruction revealed the general mesodermal rudiment of the eyeball muscles, its shape and the beginning of differentiation of each individual muscle from the mesoderm rudiment, which has a funnel-shap and covers the optic stalk. Its thickened end is directed towards the eyeball and ends in front with five small protrusions. These protrusions, as shown by studies of microspecimens of older age groups (prefetal period of IUD), are a morphological substrate for the development of individual muscles of the eyeball (Fig. 6). In addition, the mesodermal rudiment is the basis for the formation of a common tendon ring of the proximal ends of the eye muscles. The inferior oblique muscle develops from a single mesodermal rudiment and therefore has a different location and fixation points.

Simultaneously with the rudiments of the extraocular muscles, the trochlear and abductor nerves are develop and ingrown into the orbit. In the embryonic period, we noted a fairly large diameter of these nerves relative to the small thickness of the rudiments of the muscles (Fig. 7). Later, in the process of further development, there is a gradual predominance of the growth of the extraocular muscles relative to the nerves that provide their innervation.

In the cell mass of the mesenchyme of embryos of 7.0$7.5 \mathrm{~mm}$ PCL, which surrounds the eyes and mesodermal sources of the muscles, form chains of islets of intraorganic hematopoiesis and cells such as erythroblasts. Part of the islets of intraorganic hematopoiesis is separated from the adjacent mesenchyme by a number of elongated endothelial cells. In embryos of 13.0-16.0 mm PCL there is a vascular network in the orbital region (Fig. 8). It is during this period that the differentiation of the individual extraocular muscles begins.

In addition, the muscular connecting branches of extraorganic vessels grow into the eye muscles. The combination of both parts of the vascular formations occurs in the prefetuses $18.0-22.0 \mathrm{~mm}$ TCL. Thus, the vascular supply to the extraocular muscles is formed in two ways - in the form of islets of intra-organ hematopoiesis and ingrown muscle branches of extra-organ vessels. 


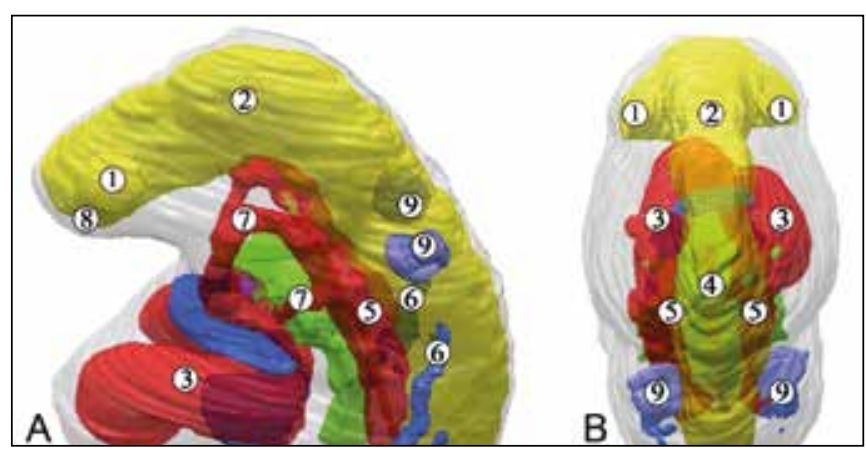

Fig. 1. 3D-reconstruction of the upper half of the human embryo $4.0 \mathrm{~mm}$ $P C L$. Left lateral $(A)$ and superior posterior (B) projections. Magn.: $x 20$ : 1 - optic vesicle; 2 - brain; 3 - heart; 4 - notochord; 5 - dorsal aorta; 6 - cardinal vein; 7 - aortic arches; 8 - frontal process; 9 - otic vesicle.

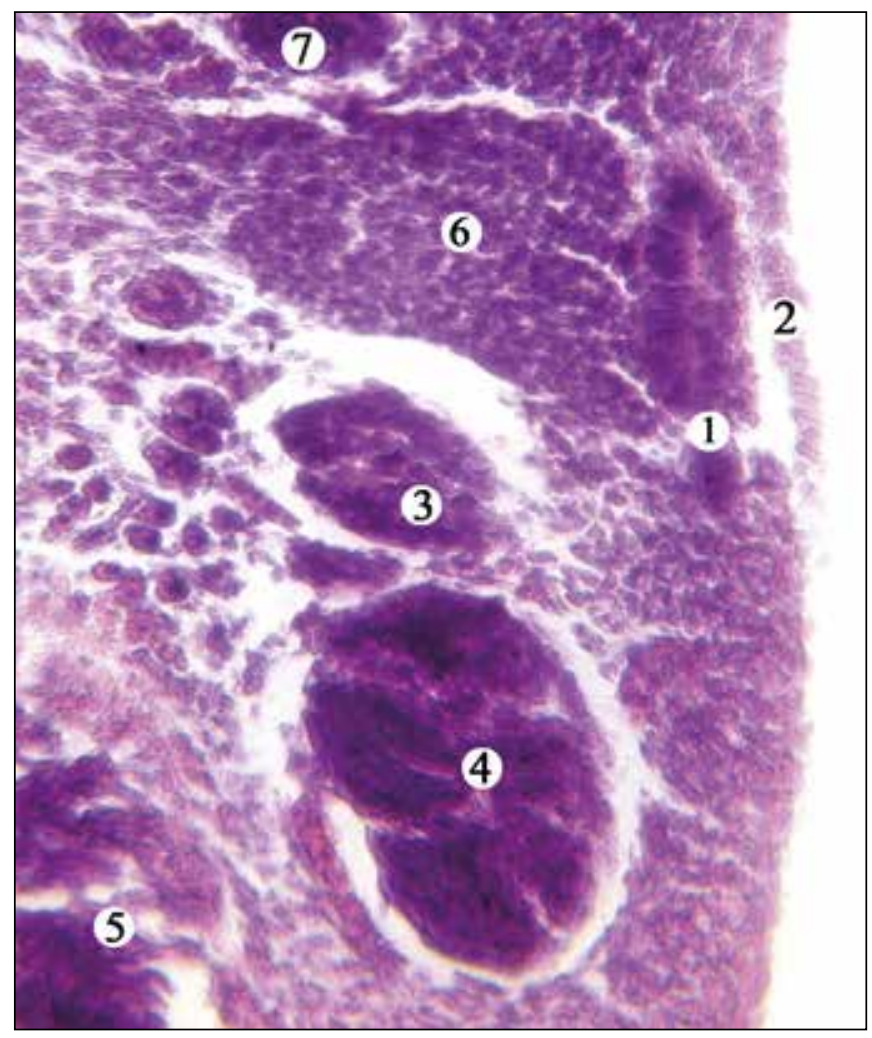

Fig. 2. Frontal histological section of the cranial end of the human embryo $3.5 \mathrm{~mm}$ PCL (3rd week of IUD) (right half). Hematoxylin and eosin staining. Microphotograph. Magn.: x200: 1 - neuroectoderm of the optic vesicle; 2 - ectodermal lens placode; 3 - VII cranial nerve; 4 - VIII cranial nerve; 5 - neuroectoderm of the midbrain; 6 - optic stalk; 7 - neuroectoderm of the telencephalon.

\section{DISCUSSION}

In our study by means of a complex of morphological methods, the development of the structures of the orbit and the organ of vision successively in the age-related dynamics of prenatal development has been studied. The method of $3 \mathrm{D}$ reconstruction of a series of consecutive histological sections of the head and microscopic examination of our material was widely used.

The majority of scientific studies of prenatal development of the human organ of visus is limited to a certain age

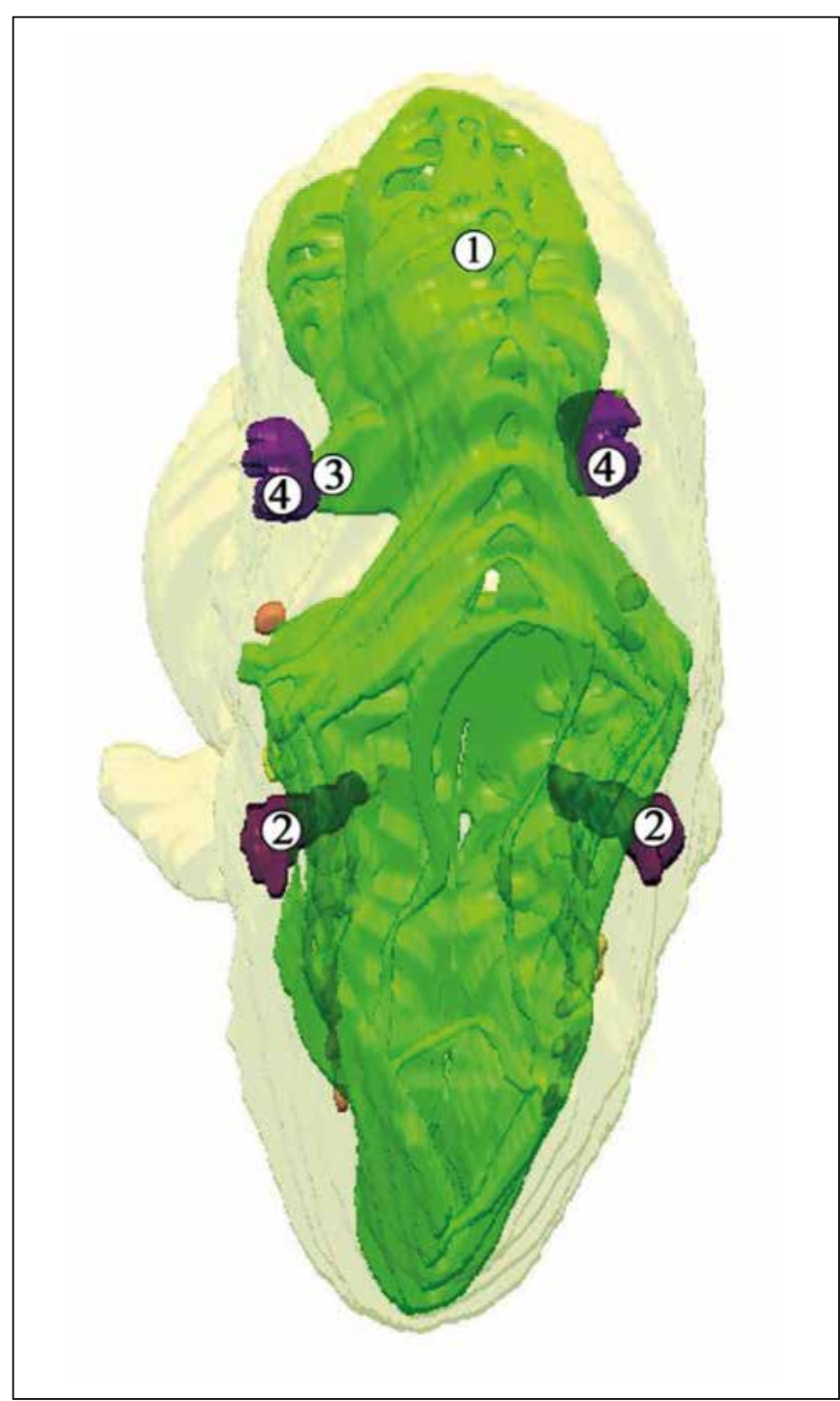

Fig. 3. 3D-reconstruction of the upper half of the human embryo $5.0 \mathrm{~mm}$ PCL. Superior posterior projection. Magn.: x20: 1 - brain; 2 - otic vesicle; 3 - optic stalk; 4 - optic cups.

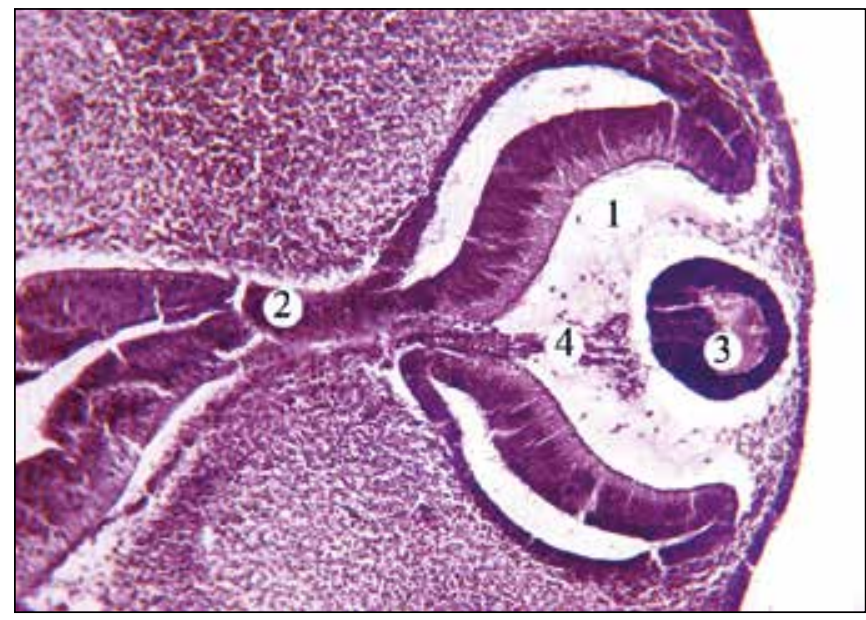

Fig. 4. Frontal section of the left cranial part of the embryo $9.0 \mathrm{~mm} \mathrm{PCL}$ (6th week of IUD). Hematoxylin and eosin staining. Microphotograph. Magn.: $x 40: 1$ - the rudiment of the eyeball; 2 - optic stulk; 3 - lens vesicle; 4 - hyaloid artery. 


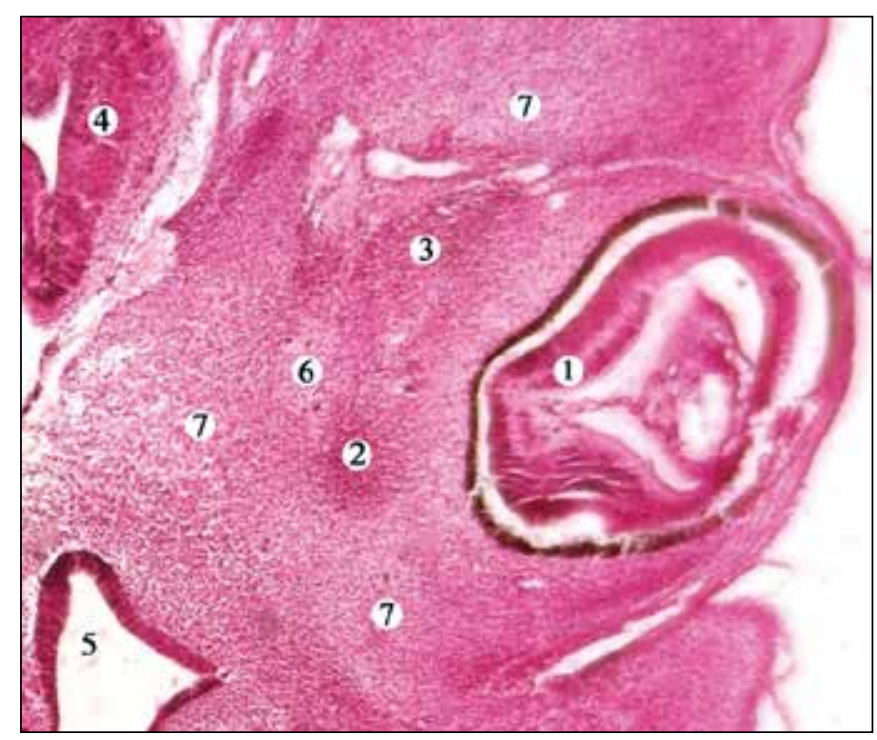

Fig. 5. Frontal section of the left side of the human embryo head $7.0 \mathrm{~mm}$ PCL. Hematoxylin and eosin staining. Photomicrograph. Magn.: x60: 1 eye; 2 - the rudiment of the lateral rectus muscle; 3 - the rudiment of the superir rectus muscle; 4 - brain; 5 - the left upper nasal conch; 6 - the rudiment of the optic nerve; 7 - mesenchyme of the head.

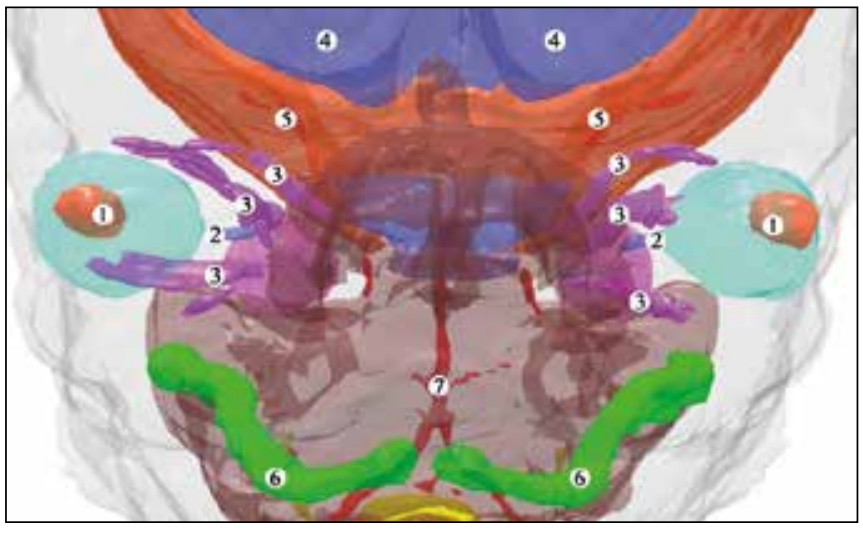

Fig. 6.3D-reconstruction of the human prefetus head $13.5 \mathrm{~mm}$ PCL. Front projection. Magn.: $\times 25: 1$ - the rudiments of the eyeballs; 2 - optic nerve; 3 -the rudiments of the extraocular muscles; 4 -hemispheres of the brain; 5 - the rudiment of skull bones; 6 - Meckel's cartilage; 7 - basilar artery.

period. We studied the development of the orbit and the eye from its source (embryos 3-4 weeks IUD), and to the beginning of the prenatal period (8th week IUD), when we can talk about the presence of all structures of the organ of visus (including blood vessels, nerves, muscles) in almost definitive topographic and anatomical relations.

The question of the time and sequence of appearance of germs of the main structures of the human organ of visus remains debatable. Thus, according to our data, the optic pits are formed in the form of depressions on both sides of the neural tube in the forebrain, even before the complete closure of the neural tube. At the beginning of the 4th week of IUD, after closing the neural tube, the optic pits form lateral optical vesicles. According to Cook C.S. [8], this process occurs on the 25th day, which corresponds to the middle of the 4 th week of IUD. Some differences in the time of appearance

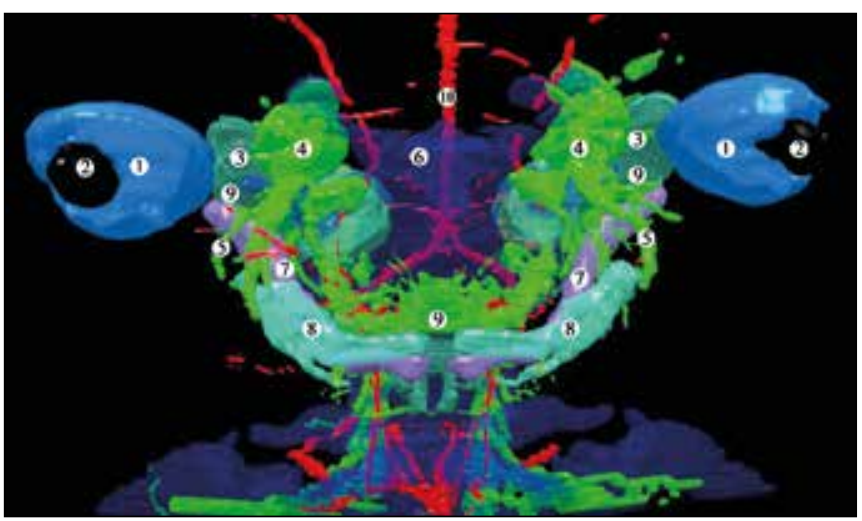

Fig. 7.3D-reconstruction of the human prefetus head $17.0 \mathrm{~mm} P C L$. Front projection. Magn.: x25:1 - eyeballs; 2 -lens; 3 - opticnerve; 4 -trigeminal nerve; 5 - facial nerve; 6 - the rudiments of skull bones; 7 - Meckel's cartilage; 8 - the rudiment of the mandible; 9 - spine; 10 - artery.

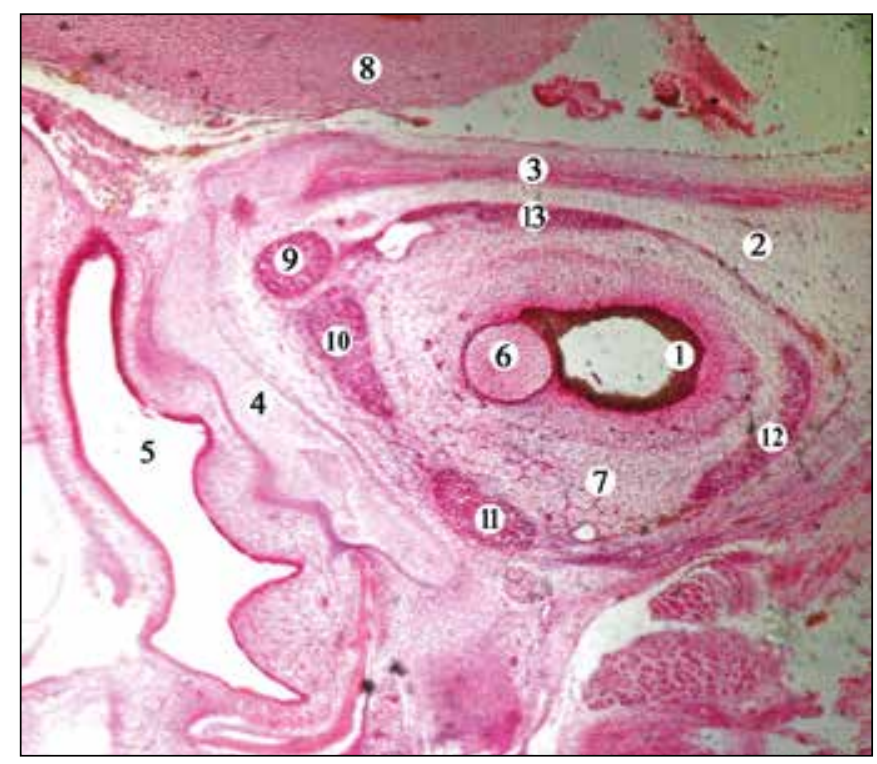

Fig. 8. Frontal section of the left half of the human embryo head 16.0 $\mathrm{mm}$ PCL. Hematoxylin and eosin staining. Photomicrograph. Magn.: x60: 1 - pigment epithelium; 2 - mesenchyme of the orbit; 3 - rudiment of the frontal bone; 4 - rudiment of the lacrimal bone; 5 - nasal cavity; 6 - optic nerve; 7 - blood vessels of the orbit; 8 - brain; 9 - superior oblique muscle; 10 - medial rectus muscle; 11 - inferior rectus muscle; 12 - lateral rectus muscle; 13 - upper rectus muscle.

of sources can be explained by an error in the methods of determining the age of the embryo. During the 4th week of IUD, the optic vesicles are initially in contact with the superficial ectoderm, but are gradually separated from it by cells originating from the neural crest and mesoderm. The cells of the neural crest and the mesoderm together form the mesenchyme, from which the connective tissue of the eyeball and the orbit develops. It is difficult to determine the origin of a certain structure - from the nerve crest or from the mesoderm, by classical morphological methods, because mesodermal cells and cells of the nerve crest have the same cytological appearance.

A number of successive processes of structural transformation of the rudiment of the organ of visus leads to the 
beginning of the formation of the optic cup and optic stalk at the 5th week of IUD, which ends with the formation of a two-layer eye cup at 7 th week of IUD [9].

The mesenchyme proliferates and migrates around the optic cup, and subsequently differentiates into the connective tissue of the orbit. The cells of the neural crest form the stroma of the cornea and endothelium, the choroid plexus and melanocytes, the ciliary muscle, most of the sclera, connective tissue and meningeal tunic of the optic nerve, connective tissue of the eyelids, conjunctiva and orbit. There is no doubt that the vascular endothelium and striated muscles are formed from the mesoderm [10]. We do not agree with Mann I. [11] that the external muscles of the eye develops gradually - first behind, at the apex of the orbit, from where they gradually grow frontward. We agree with the statement of Barishak Y.R. [10], Sevel D. [12] on the almost simultaneous development of extraocular muscles and their structural components. According to them, the muscles innervated by the III pair of cranial nerves originate from the I pair of somites on about the 26th day of IUD. The lateral rectus muscle, innervated by the VI pair of cranial nerves, develops from the mesenchyme of the maxillar and mandibular regions on about day 27 of IUD. The anterior oblique muscle, innervated by the IV pair of cranial nerves, originates from the II pair of somites on day 29 of IUD. This is broadly consistent with our results on the sources of muscles in embryos in the middle of the 5th week of IUD.

\section{CONCLUSIONS}

1. The rudiments of the organ of vision (lens placodes) appear in the 4rd week of IUD. At the 5th week of IUD as a result of gradual intussusception of crystalline placodes into the adjacent mesenchyme, lens pit are formed, and then - lens vesicles.

2. Rudiments of the extraocular muscles (except for the inferior oblique muscle) was detected at the end of the 5 th week of IUD. The rudiment of the inferior oblique muscle develops from a single mesodermal islet located in the mesenchyme medially and below the eyeball. Simultaneously with the rudiments of the extraokular muscles, the trochlear and abductor nerves are develop and ingrown into the orbit.

3. The development of blood vessels of the orbit occurs from two sources - from the islands of local angiogenesis, which begins in the 5th week of IUD, and from extraorganic vessels, which can be traced in the form of a vascular network at the end of the 6th week of IUD. The combination of both sources is observed at the end of the 7 th week of IUD.

4. The embryonic period of ontogenesis is the first critical period in the development of the human orbit, due to the formation of the muscles, nerve and vascular structures.

\section{REFERENCES}

1. Tawfik H.A., Dutton J.J. Embryologic and Fetal Development of the Human Orbit. Ophthalmic Plastic \& Reconstructive Surgery. 2018;34(5):405-421.
2. Yamaguchi K. Development of the human oculomotor nuclear complex: Somatic nuclei. Annals of Anatomy-Anatomischer Anzeiger. 2014; 196(6):394-401.

3. Kononova N.E., Somov E.E. K otsenke rezultatov letchenia detey, stradauchih ambliopiey, svazanoy s sodrujestvenym kosoglaziem. [To evaluate the results of treatment of children with amblyopia associated with friendly strabismus]. Pediatrician. 2017; 8(5):25-29. (in Ukrainian).

4. Manton N.D. Skeletal Muscle and Peripheral Nerves. In Keeling's Fetal and Neonatal Pathology. Springer, Cham. 2015: 767-787.

5. Rao H.L., Pradhan Z.S., Weinreb R.N. et al. Relationship of optic nerve structure and function to peripapillary vessel density measurements of optical coherence tomography angiography in glaucoma. Journal of Glaucoma. 2017; 26(6): 548-554.

6. Salman M.S., Klassen S.F., Clark I.H. Congenital oculomotor nerve paresis with isolated cyclic pupillary spasms. Journal of Neuro-Ophthalmology. 2015;35(4): 371-373.

7. Burns N.S., lyer R.S., Robinson A.J., Chapman T. Diagnostic imaging of fetal and pediatric orbital abnormalities. American Journal of Roentgenology. 2013;201(6):W797-W808.

8. Cook C.S. Prenatal development of the eye and its adnexa. Foundations of clinical ophthalmology. 1994; 1: 1-93.

9. Barber A.N. Embryology of the human eye. St. Louis: CV Mosby Co. 1955: 193-195.

10. Barishak Y.R. Embryology of the Eye and Its Adnexae. Developments in ophthalmology. 1992; 24: 1-142.

11. Mann I. Development of the human eye, Grune \& Stratton, New York. $1964 .$.

12. Sevel D. A reappraisal of the origin of human extraocular muscles. Ophthalmology. 1981; 88(12): 1330-1338.

\section{ORCID and contributionship:}

Olexandr V. Tsyhykalo: 0000-0003-2302-426X ${ }^{A, B, D}$

Igor Yu. Oliinyk: 0000-0002-6221-8078 ${ }^{B, C, F}$

Nataliya Ya. Kozariichuk: 0000-0002-8884-507X ${ }^{C, D, F}$

Larysa Ya. Fedoniuk: 0000-0003-4910-6888 E,F

Lyudmila V. Fomina: 0000-0002-1695-3442 ${ }^{\mathrm{E}}$

Olha L. Ocheretna: 0000-0001-7895-2931 ${ }^{F}$

Viktoriia V. Piliponova: 0000-0002-5465-5809 ${ }^{F}$

\section{Conflict of interest:}

The Author declare no conflict of interest.

\section{CORRESPONDING AUTHOR \\ Olexandr V. Tsyhykalo \\ Bukovinian State Medical University \\ 4 Teatralva Sq., 58001, Chernivtsi, Ukraine \\ tel: +380990737261 \\ e-mail: tsyhykalo@icloud.com}

Received: 21.08 .2020

Accepted: 05.01.2021
A - Work concept and design, B - Data collection and analysis, C - Responsibility for statistical analysis, D-Writing the article, $\mathbf{E}$-Critical review, $\mathbf{F}$ - Final approval of the article 